\title{
Temperature effects on the mid-and far-infrared spectra of olivine particles ${ }^{\star}$
}

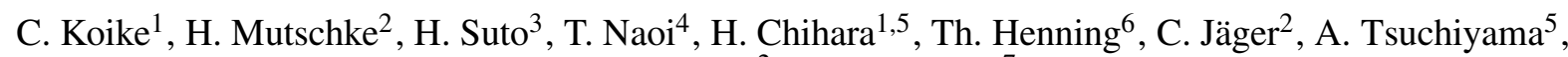 \\ J. Dorschner ${ }^{2}$, and H. Okuda ${ }^{7}$
}

\author{
1 Kyoto Pharmaceutical University, Yamashina, Kyoto 607-8412, Japan \\ e-mail: koike@mb. kyoto-phu.ac.jp \\ 2 Astrophysical Institute and University Observatory, Friedrich Schiller University Jena, Schillergäßchen 2-3, 07743 Jena, Germany \\ 3 Subaru Telescope, National Astronomical Observatory of Japan, Hilo, Hawaii, USA \\ 4 Department of Earth \& Planetary Science, University of Tokyo, Hongo, Tokyo 133-0033, Japan \\ 5 Department of Earth and Space Science, Osaka University, Toyonaka 560-0043, Japan \\ ${ }^{6}$ Max Planck Institute for Astronomy, Königstuhl 17, 69117 Heidelberg, Germany \\ 7 Gunma Astronomical Observatory, Agatsuma, Gunma 377-0702, Japan
}

Received 18 April 2005 / Accepted 7 November 2005

\section{ABSTRACT}

The absorption spectra of the olivine particles of different $\mathrm{Mg} / \mathrm{Fe}$ content were measured in the infrared spectral region between 5 and $100 \mu \mathrm{m}$, while the particles were continuously cooled down to $10 \mathrm{~K}$. Measurements independently carried out on different samples of synthetic forsterite, natural olivine, and synthetic fayalite at laboratories in Kyoto and Jena. The positions of the olivine infrared bands were measured for these samples in detail at up to seven individual temperatures in the interval between $300 \mathrm{~K}$ and $10 \mathrm{~K}$. According to the different widths of the olivine bands in different wavelength regions, spectral resolutions of $2,1,0.5,0.25,0.2$, and $0.125 \mathrm{~cm}^{-1}$ were used in order to measure the band positions with high accuracy. While in general the band positions and their temperature-dependent shift agree very well for the Kyoto and Jena samples, the positions of some very strong bands differ, which is probably a consequence of different particle shapes. For the two long-wavelength forsterite bands at 49 and $69 \mu \mathrm{m}$, the sharpening and strengthening of the bands were quantified. The widths of these bands differ for the Kyoto and Jena samples, which is discussed in terms of different crystal quality and particle coagulation of the samples. Our new data can be used to derive dust temperatures from the observed peak positions for crystalline silicate dust in circumstellar regions.

Key words. stars: circumstellar matter

\section{Introduction}

The many interesting infrared spectra of comets, young stellar objects, and oxygen-rich dust in outflows and discs associated with late-type stars and planetary nebulae have been observed with the Infrared Space Observatory (ISO) (e.g. Waters et al. 1996; Waelkens et al. 1996; Crovisier et al. 1997; Malfait et al. 1999). They reveal the presence of crystalline silicate dust in many of these sources, especially of silicates of the olivine crystal class, the composition of which is characterized by the general formula $[\mathrm{Mg}, \mathrm{Fe}, \ldots]_{2} \mathrm{SiO}_{4}$ (see Henning 2003; and Colangeli et al. 2003, for reviews). The lattice vibrations of these silicates lead to infrared emission bands in

* Jena data (4 tables) are only available in electronic form at the CDS via anonymous ftp to cdsarc.u-strasbg.fr (130.79.128.5) or via

http://cdsweb.u-strasbg.fr/cgi-bin/qcat?J/A+A/449/583 the range of about 10 to $70 \mu \mathrm{m}$, and they have been detected by ISO and have been identified by comparison with laboratory data (e.g. Jäger et al. 1998). Simple spectral fits indicate that a large amount of the optically thin silicate emission often originates from particles in the temperature range of 50 to $100 \mathrm{~K}$. However, owing to the lack of laboratory data, both simple blackbody fits and radiative transfer models of these environments applied optical dust data measured at room temperature. It is well known from previous studies that the dust temperature strongly affects the frequency, bandwidth, and band structure of vibrational bands of solids (see, e.g. Mitra 1985). Since the lattice potentials are anharmonic to some degree, the mean interatomic distances and forces depend on the level of vibrational excitation. Consequently, transitions between higher levels are typically less energetic than transitions between lower ones. This causes both a shift in the corresponding bands to lower energy at higher temperature and a broadening, because at higher temperature transitions between more states are contributing. 
Such temperature effects on infrared spectra by several groups from an astronomical perspective have been investigated. Zaikowski et al. (1975) measured the $10 \mu \mathrm{m}$ band of forsterite and hydrous silicates at $4 \mathrm{~K}$. For the mid-infrared spectra of forsterite and pyroxene at $80 \mathrm{~K}$, Day (1976) showed that the intensity and sharpness of the bands increased and that some peaks shifted toward shorter wavelength. Henning \& Mutschke (1997) measured the reflectance and absorption spectra of a number of cosmic dust analogues up to far-infrared wavelengths and derived optical constants at temperatures down to $10 \mathrm{~K}$. But, only one crystalline silicate of pyroxene stoichiometry (bronzite) was included in this study. Mennella et al. (1998) investigated the temperature effects on the spectral features of carbonaceous materials, amorphous silicates, and two crystalline silicates (natural olivines with iron contents of $6 \%$ and $90 \%$, respectively), at wavelengths from $20 \mu \mathrm{m}$ to the millimeter region in the temperature range from 295 to $24 \mathrm{~K}$. They report that the absorption bands of the crystalline silicates shift to shorter wavelengths at low temperatures and that the farinfrared continuum emissivities of all the samples decrease in this temperature range ${ }^{1}$. In these studies, however, no quantitative discussion of the variation in the individual absorption bands was satisfactorily given, because of uncertainties due to the low spectral resolution of the measurements.

Bowey et al. (2001) first measured the infrared band positions at $3.5 \mathrm{~K}$ compared to room temperature at a high resolution of $0.25 \mathrm{~cm}^{-1}$. These measurements demonstrate for a number of natural silicate minerals and synthetic forsterite $\left(\mathrm{Mg}_{2} \mathrm{SiO}_{4}\right)$ that the temperature significantly affects the band positions and that some of the bands, such as the $69 \mu \mathrm{m}$ band of forsterite, may be used as temperature indicators (Bowey et al. 2002). For other bands, especially of pyroxenes and hydrous silicates, the shift has to be considered more complex.

However, as mentioned by Bowey et al. (2002), the temperature resolution of laboratory measurements is not yet sufficient enough for an accurate determination of peak positions and band widths at a particular dust temperature, which is needed for comparison with observed spectra. In this study, we attempt to quantitatively investigate the temperature dependence of these data for pure and high-quality synthetic crystalline silicates. In a first step, Chihara et al. (2001) measured the temperature effect on the bands of synthetic crystalline olivines and pyroxenes cooled down to the liquid-helium temperature $(4.2 \mathrm{~K})$. In this paper, we extend these data by presenting band positions and bandwidths (for some bands) derived from absorption spectra of olivine particles with different iron content, measured at various temperatures while cooling down the samples to $8 \mathrm{~K}$. The spectra were measured in the mid-and far-infrared regions using Fourier transform spectrometers at a high spectral resolution in two laboratories in Kyoto and Jena.

Our experimental data can be obtained from the web-sites (http://www.kyoto-phu.ac.jp/labo/butsuri/ and http://www . astro.uni-jena.de/Laboratory/OCDB). The data are also available at the CDS.

\footnotetext{
${ }^{1}$ This continuum emissivity - for other studies see Agladze et al. (1996) and Boudet et al. (2005) - is not of major relevance for the vibrational bands investigated in this paper.
}

\section{Measurements}

The olivine samples we used were synthetic forsterite $\mathrm{Mg}_{2} \mathrm{SiO}_{4}$ (Fo), natural olivine from San Carlos, and synthetic fayalite $\mathrm{Fe}_{2} \mathrm{SiO}_{4}(\mathrm{Fa})$, which were produced and obtained by the Kyoto and Jena groups independently. The composition of the natural olivine has been determined by EPMA for the Kyoto sample to be $\mathrm{Fo}_{90.7}$, i.e., $90.7 \%$ magnesium and $9.3 \%$ iron as the metal cations. For the Jena sample, the composition determined by EDX was Fo92.4 40.5 .

The Kyoto Fo and Fa samples were synthesized by the Czochralski method and the floating-zone method, respectively, as reported by Koike et al. (2003). The Jena Fo and Fa samples have been produced by melting in Pt crucibles and by the scull method, respectively, as reported by Fabian et al. (2001).

The olivine crystals were crushed down in an agate and/or alumina mortar (for the Kyoto samples) and in a Sialon ball mill (for the Jena samples) to produce fine grains with smaller sizes than $1 \mu \mathrm{m}$. For measurement of the Jena samples at wavelengths up to $25 \mu \mathrm{m}, 0.4 \mathrm{mg}$ of each of the olivine particle samples were embedded in $\mathrm{KBr}$ pellets with a $13 \mathrm{~mm}$ diameter and $200 \mathrm{mg}$ weight ( $0.2 \%$ of weight). For the Kyoto samples, the olivine particles were embedded in $\mathrm{KBr}$ at a mass ratio of $0.2-0.3 \%$ weight. For measurements at longer wavelengths, in the Jena lab polyethylene (PE) pellets with the same size and mass as the $\mathrm{KBr}$ pellets, containing either $0.4 \mathrm{mg}(0.2 \%$ weight $)$ or $2 \mathrm{mg}$ ( $1 \%$ weight, for measurement of the weaker bands at wavelengths larger than $40 \mu \mathrm{m}$ ) of particles were prepared. In the Kyoto lab, PE sheets with diameters ranging from about 15 to $20 \mathrm{~mm}$ and thicknesses of about $1 \mathrm{~mm}$ were prepared. Again, two types of sheets with lower $(0.42-1.1 \%$ weight $)$ and higher (1-5.9\% weight) ratio of sample mass to PE mass were prepared in order to be able to take measurements in wavelength regions with stronger and weaker bands, respectively.

For sample cooling, cryostats equipped with KRS5 and polyethylene infrared optical windows were adopted. In the case of the Kyoto samples, the cryostat was cooled by a closedcycle He cryostat, whereas in the case of the Jena samples, cooling by liquid-helium flow was applied. The Jena setup has already been described by Henning \& Mutschke (1997). In both cases, the sample temperature was controlled by an appropriate temperature controller (Lakeshore or Cryovac) using an ohmic heater and a silicon diode temperature sensor on the sample stage. The sample holders were able to hold two samples, thus allowing between the sample and a blank reference sample to be switched without warming up.

For the Kyoto samples, the transmittances $T$ of the $\mathrm{KBr}$ pellets and PE sheets (relative to the transmittances of the respective reference samples) were measured generally at temperatures of 295, 200, 150,100, 50, 20 and $10 \mathrm{~K}$ with Nicolet Nexus 670 and 6700 FTIR spectrometers from $4000 \mathrm{~cm}^{-1}$ to $50 \mathrm{~cm}^{-1}$. The resolution was $1,0.5,0.25$, and $0.125 \mathrm{~cm}^{-1}$ according to the need to resolve the narrow features. The Jena samples were measured at 295, 200, 100, and $10 \mathrm{~K}$ with a Bruker 113v FTIR spectrometer at resolutions of $2 \mathrm{~cm}^{-1}$ and $0.2 \mathrm{~cm}^{-1}$ (at longer wavelengths than $40 \mu \mathrm{m}$ ). 
The mass absorption coefficient $\kappa(\lambda)\left(\mathrm{cm}^{2} / \mathrm{g}\right)$ is derived from the transmission spectra $T(\lambda)$ of the samples according to the following equation:

$\kappa=\frac{S}{M} \ln \frac{1}{T}$.

Here, $S$ is the surface area of a $\mathrm{KBr}$ pellet or polyethylene sheet that includes sample powders, and $M$ is the mass of the sample embedded in the sample pellet or sheet.

\section{Results}

\subsection{Overview of the spectra}

The absorption spectra of the olivine particles obtained in the Kyoto lab in the mid- and far-infrared region are shown in Figs. 1-3. The effect of the temperature is noticeable for all spectra, particularly in the far-infrared region, where all bands became stronger and narrower, and shifted to shorter wavelengths at low temperature. In the mid-infrared, the effect of the temperature is more difficult to realize. Figure 4 shows that the relative shifts $\Delta \lambda / \lambda(T)$ are at least weaker by a factor of two for the bands in the $10 \mu \mathrm{m}$ region than for bands at larger wavelengths. This result is reasonable, because the tetrahedral Si-O bond lengths and bond angles are relatively incompressible in comparison with the spacing between the oxygen and metal ions and intertetrahedral oxygen atoms. Consequently, there is little variation in the wavelength of the $10-\mu \mathrm{m}$ stretching and $20-\mu \mathrm{m}$ bending features with temperature (Day 1976; Bowey et al. 2001). In contrast, the volumes of the divalent-cation sites are more compressible, therefore, the wavelengths of the farinfrared peaks are shifted much more to shorter wavelengths. Furthermore, Fig. 4 demonstrates that for most of the forsterite bands, the peak shift per temperature interval is stronger at high temperatures than at low temperatures $(<100 \mathrm{~K})$. This tendency is seen for most of the band positions of all three materials (see Figs. 5-7), especially for the weaker bands where shape effects are less important. Of course, this result is the most important for the use of band positions as temperature indicators (see Sect. 4.2) and has not been well established prior to this work.

\subsection{Forsterite}

The spectra of Kyoto Fo are shown in Fig. 1. The intensity and width of each band increased and decreased, respectively, as temperature decreased, and new bands appeared at around 32.5 and $36 \mu \mathrm{m}$. Furthermore, the peak positions of the bands shifted to shorter wavelengths as temperature decreased. These tendencies are significant in the far-infrared region, while the spectra in the $10 \mu \mathrm{m}$ region were not changed much after cooling to $8 \mathrm{~K}$. In particular, the bands at 33,49 , and $69 \mu \mathrm{m}$ show significant changes in the peak intensity and peak position. After cooling down to $8 \mathrm{~K}$, the strong peak at $33.8 \mu \mathrm{m}$ was shifted to $33.6 \mu \mathrm{m}$, and a new peak and a new double band appeared at 32.5 and around $36 \mu \mathrm{m}$, respectively. The new double peaks are due to the band splitting of the $36.4 \mu \mathrm{m}$ band, and are located at 35.8 and $36.1 \mu \mathrm{m}$ as shown in Fig. 1. These double peaks can also be a good indicator of dust temperature as suggested by Bowey et al. (2001). The peak intensity of the 33, 49, and $69 \mu \mathrm{m}$ bands at $8 \mathrm{~K}$ increased compared to room temperature by factors of about 1.5, 2.0, and 2.8, respectively. The peak position of each band (read from the spectra by eye) is clearly shifted to a shorter wavelength with cooling, and can also be used as an indicator of dust temperature (see Fig. 5).

In Fig. 5, the data measured in Jena are also plotted (open symbols and dotted lines). Most of the data of the Kyoto and Jena samples agree well with each other. However, for the strong peaks at 23 and $33 \mu \mathrm{m}$, the peak positions for the Kyoto and Jena samples differ by about $0.5 \mu \mathrm{m}$. For some other strong bands such as those at $10 \mu \mathrm{m}, 11.2 \mu \mathrm{m}$, and $19.5 \mu \mathrm{m}$, the peak positions of the Jena sample are also shifted to the short wavelength side compared to those of the Kyoto sample. These differences will be discussed in Sect. 4.1. The peak positions of the forsterite bands are listed in Table 1.

\subsection{Fayalite}

The spectra of the Kyoto fayalite are shown in Fig. 2. The fayalite bands changed significantly while cooling the sample down to $8 \mathrm{~K}$. The intensity and width of each band increased and decreased, respectively, as temperature decreased, and new bands appeared at around 39, 53, and $56 \mu \mathrm{m}$. In particular, the spectra in the wavelength range from 50 to $60 \mu \mathrm{m}$ changed significantly after cooling down to $8 \mathrm{~K}$. Furthermore, the peak positions of the bands (read from the spectra by eye) shifted to shorter wavelengths as temperature decreased, as shown in Fig. 6, which also plots the Jena data. The data of the Kyoto and Jena samples almost all agreed. The differences in the peak positions of the strong bands, which were noticed for Fo and natural olivine (Figs. 5 and 7), were not observed (see Sect. 4.1). If fayalite dust particles exist in circumstellar environments, each peak position may become a good indicator of the dust temperature, as shown in Fig. 6.

Hofmeister (1997) reported detecting of two bands at $86 \mathrm{~cm}^{-1}(116 \mu \mathrm{m})$ and $106 \mathrm{~cm}^{-1}(94.3 \mu \mathrm{m})$ in reflection spectra of synthetic fayalite, as well as in absorption spectra of both bulk single crystals and very thick powder samples. While this detection was confirmed neither for powder samples by Mennella et al. (1998) and Koike et al. (2003) nor for reflectance measurements by Fabian et al. (2001) and Suto et al. (2002), recently Posch et al. (2005) again found two bands at 93-94 $\mu \mathrm{m}$ and $110 \mu \mathrm{m}$ in spectra of fayalite thin sections $(0.8 \mathrm{~mm}$ thickness) at low temperatures (200 to $40 \mathrm{~K})$. Therefore, we tried to detect these two bands at low temperature for the Kyoto sample. For this purpose, we also measured PE sheets of high mass ratio of samples ( $3.3 \%$ weight) at temperatures down to $10 \mathrm{~K}$. These spectra are plotted as dotted lines in Fig. 2. They do not show any bands in the $80-110 \mu \mathrm{m}$ spectral range. The reason may be that the sample weight ratio was insufficient for a detection at our $\mathrm{S} / \mathrm{N}$ ratio. We conclude that these bands, if present in the Kyoto fayalite, are similarly weak as in the Jena fayalite used by Posch et al. (2005). 


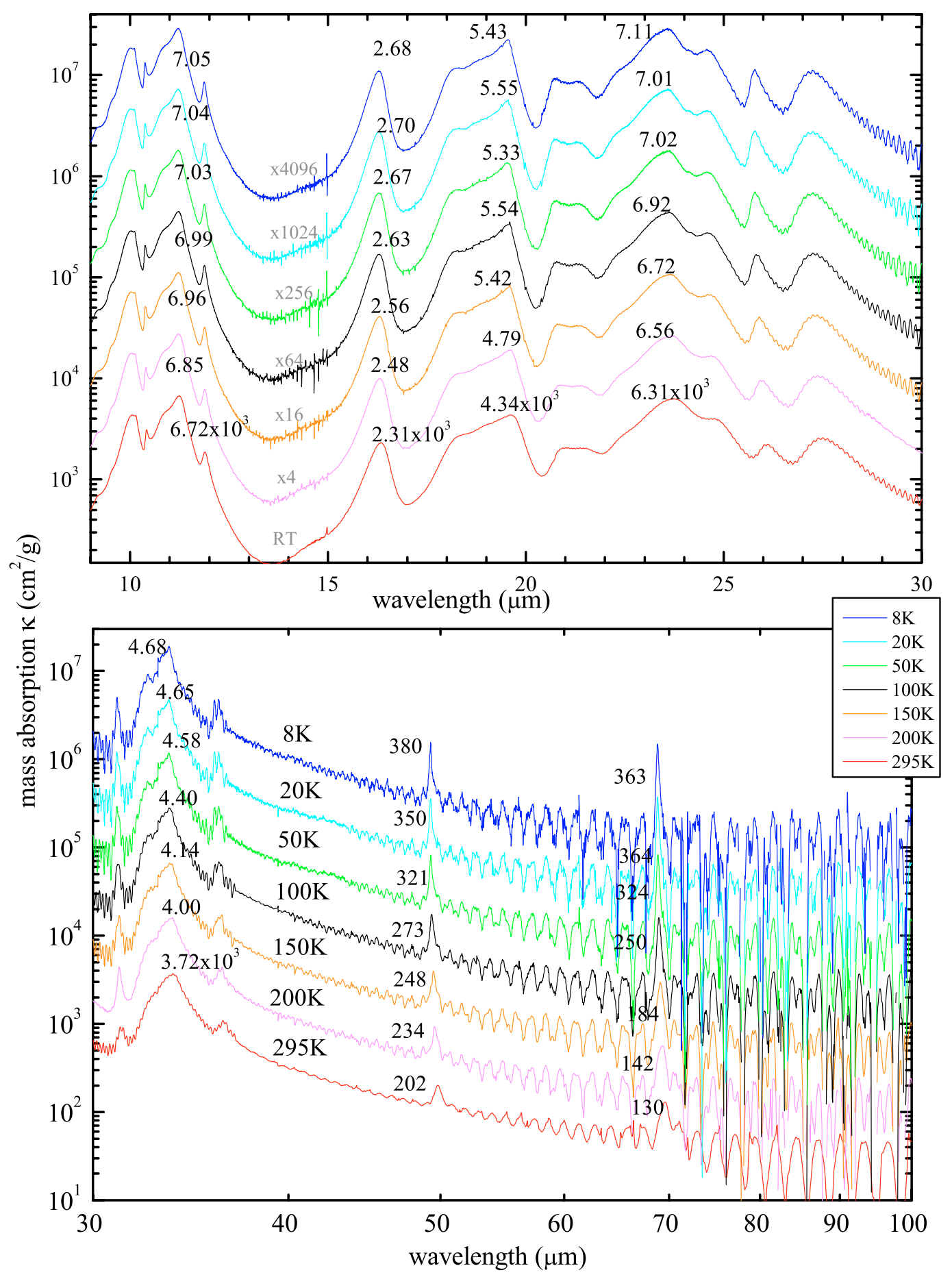

Fig. 1. The spectra of Kyoto forsterite at room temperature 200, 150, 100, 50, 20, and $8 \mathrm{~K}$. For clarity, each spectrum is multiplied by the factor indicated in gray in the upper plot. The numbers are mass absorption coefficients $\kappa$ at strong peaks.

\subsection{Natural olivine Fogo.7}

The spectra of the Kyoto sample of natural olivine Fo90.7 from San Carlos are shown in Fig. 3. The spectra of Fo90.7 did not change as significantly with temperature as the ones of the end members Fo and Fa. But the intensity of bands at about 19, 21, 24,25 , and $26 \mu \mathrm{m}$ became stronger during cooling down to $9 \mathrm{~K}$. In Fig. 7, we plot the peak positions found for both the Kyoto and Jena (open symbols and dotted lines) samples. Although the data of the Kyoto and Jena samples in general agree well with each other, the peak positions are about $0.5 \mu \mathrm{m}$ different for the strong bands at 19 and $23 \mu \mathrm{m}$, similar to the forsterite data (see Sect. 4.1).

As for the 50 and $70 \mu \mathrm{m}$ bands, large fringes exist in the farinfrared spectra for both the Kyoto and Jena measurements, in which the spectral resolutions were $0.125 \mathrm{~cm}^{-1}$ and $0.2 \mathrm{~cm}^{-1}$, respectively. As a result, the $50 \mu \mathrm{m}$ position was only detected at $10 \mathrm{~K}$ for the Jena sample, and the $70 \mu \mathrm{m}$ position 


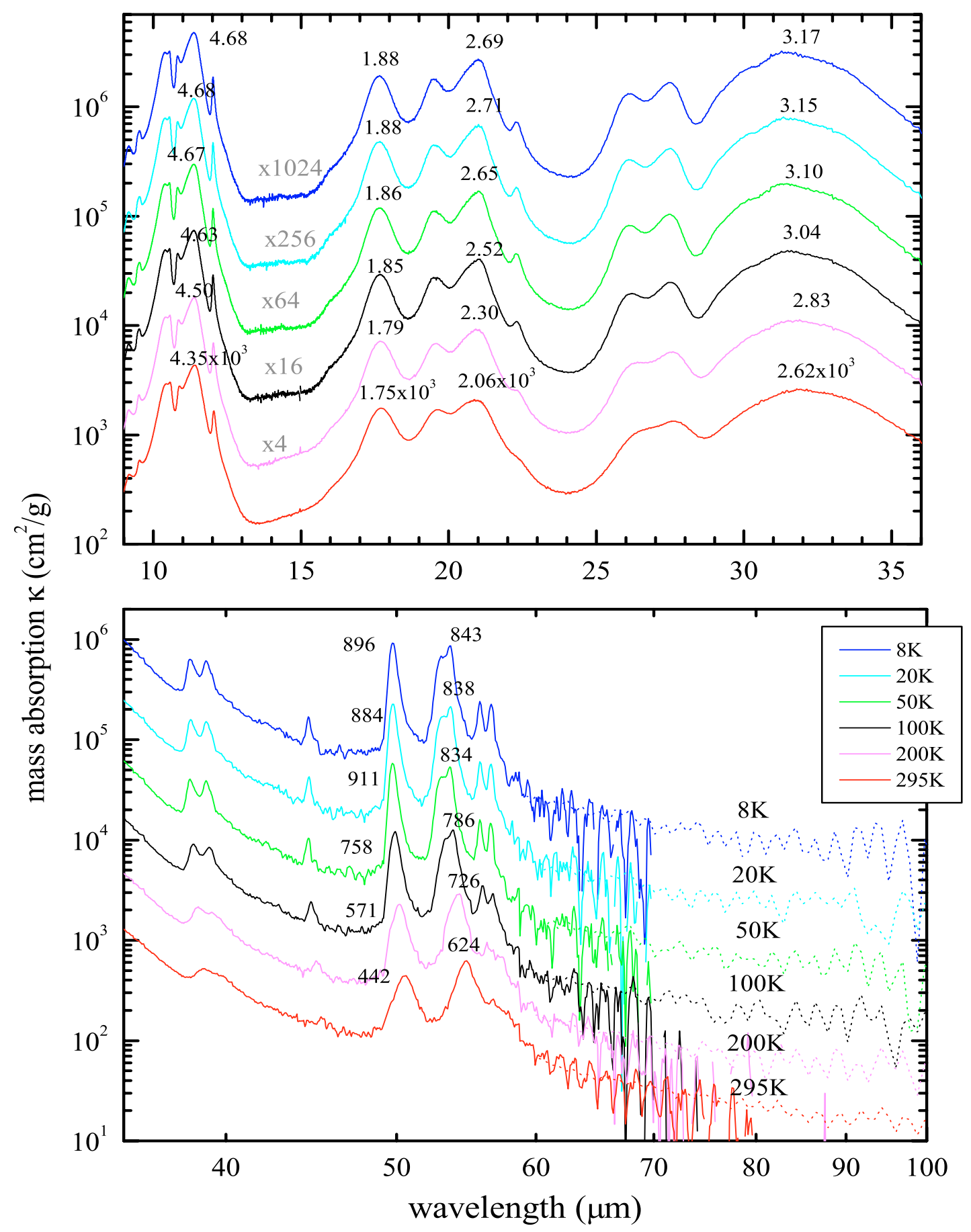

Fig. 2. The spectra of Kyoto fayalite at room temperature 200, 150, 100, 50, 20, and $8 \mathrm{~K}$. For longer wavelengths than about $80 \mu \mathrm{m}$, the measured and corrected spectra are shown as dotted lines (details in text). For clarity, each spectrum is multiplied by the factor indicated in gray in the upper plot. The numbers are mass absorption coefficients $\kappa$ at strong peaks.

for each temperature was not clearly detected for the Kyoto sample. In order to clarify the peak positions for the Kyoto sample, a denser PE sheet containing 5.4\% weight as above of olivine (F6) was prepared in addition to the medium dense sheet (F4, 1\% weight). But, due to strong fringes the peak positions were again difficult to identify. The far infrared spectra shown in Fig. 3 were measured on the dense sheet (F6). In Fig. 7, the peak positions at 50 and $70 \mu \mathrm{m}$ for both the medium and dense sheets were plotted. The positions read by eye and by Lorentzian fit have large errors due to the strong fringes. The errors by Lorentzian fit are within about $0.5 \mu \mathrm{m}$ for $295 \mathrm{~K}$ and $200 \mathrm{~K}$ and within about $0.3-0.2 \mu \mathrm{m}$ for 100,50 , 20 , and $10 \mathrm{~K}$.

In general, the $70 \mu \mathrm{m}$ band also became stronger and sharper for all the samples as the sample was cooled to a low temperature. However, the position shifted by only about $1 \mu \mathrm{m}$ to shorter wavelengths. The data by Bowey et al. (2001) at room temperature and $3.5 \mathrm{~K}$, which are also plotted in Fig. 7, 


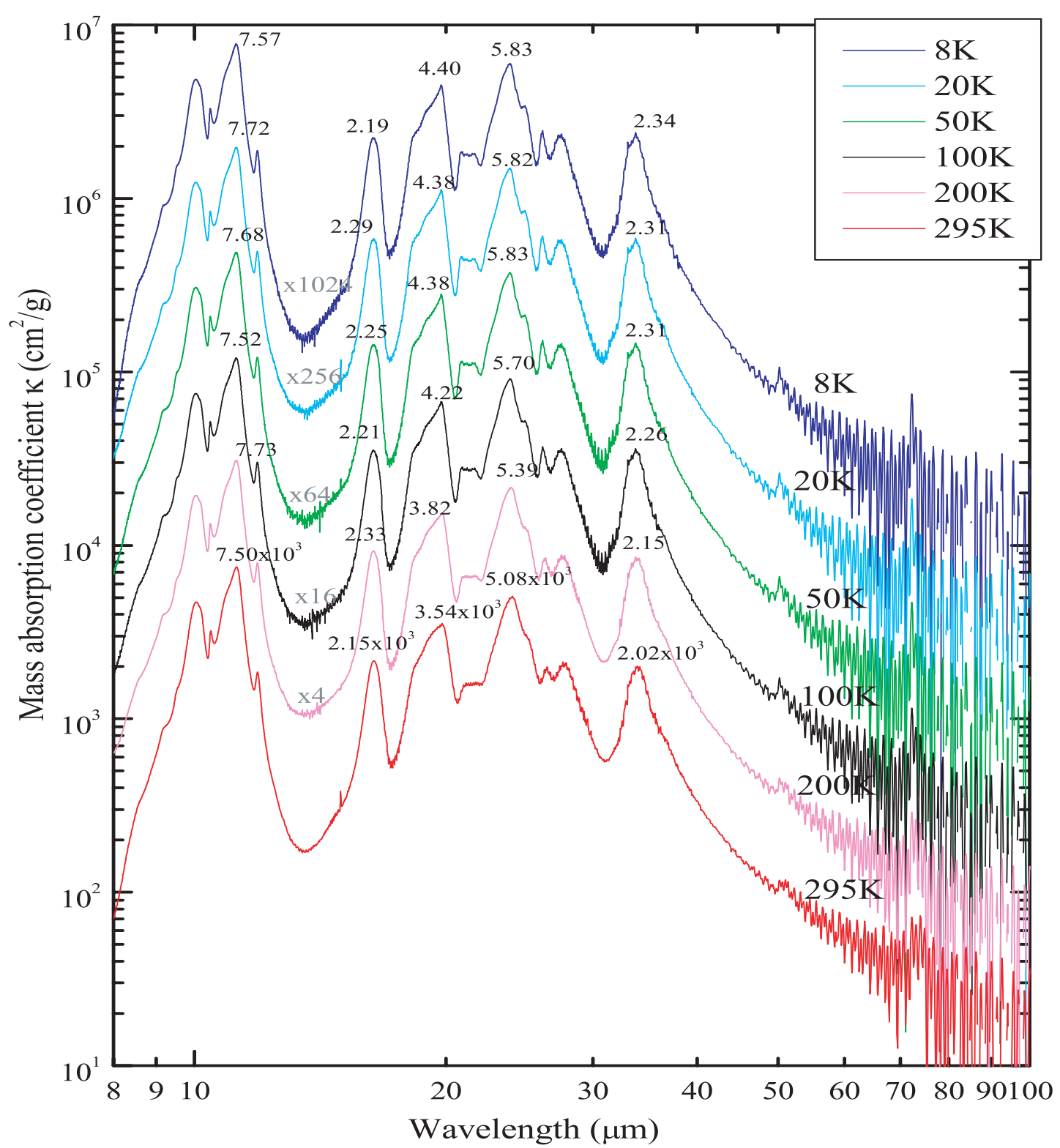

Fig. 3. The spectra of natural olivine Fo ${ }_{90.7}$ (from San Carlos) at room temperature 200, 150, 100, 50, 20, and 8 K. For clarity, each spectrum is multiplied by the factor indicated in gray in the plot. The numbers are mass absorption coefficients $\kappa$ at strong peaks.

agree well with the Kyoto data, especially sample F6 (dense sheet). The peak positions of sample F4 (medium sheet) and the Jena data are at a shorter wavelength by about $0.5 \mu \mathrm{m}$. The differences among even the Kyoto data may be due to particle aggregation and also due to particle composition, because the fine particles for the F4 and F6 sheet were produced by crushing different samples of olivine grains (composition for sample F6 not measured). In general, natural olivine may show some variability in the composition from crystal to crystal and even inside one crystal.

\section{Discussions}

\subsection{Shape effects on spectra}

As mentioned in the previous section, some of the peak positions obtained for the Jena Fo and olivine samples are at a shorter wavelength than those measured for the Kyoto samples (see Figs. 5 and 7). This is especially true for the positions of the stronger peaks. One of the materials for which the effect is seen, the natural olivine, has the same origin (San Carlos) in Kyoto and Jena. Therefore we assume that the shift is not caused by differences in the material composition or crystallinity. Furthermore, the effect occurs for both $\mathrm{KBr}$ and PE embedding.

While there are differences in the PE embedding technique in Kyoto and Jena, the $\mathrm{KBr}$ embedding is similar, so that we conclude that the effect is not due to different embedding of the samples. Instead we assume that it is related to differences in the grain shape that are caused by the use of different crushing techniques (Sialon ball mill vs. crushing by hand in an agate mortar). For the fayalite spectra this behavior is seen at a much lower level (Fig. 6), but fayalite particles are soft and can be 

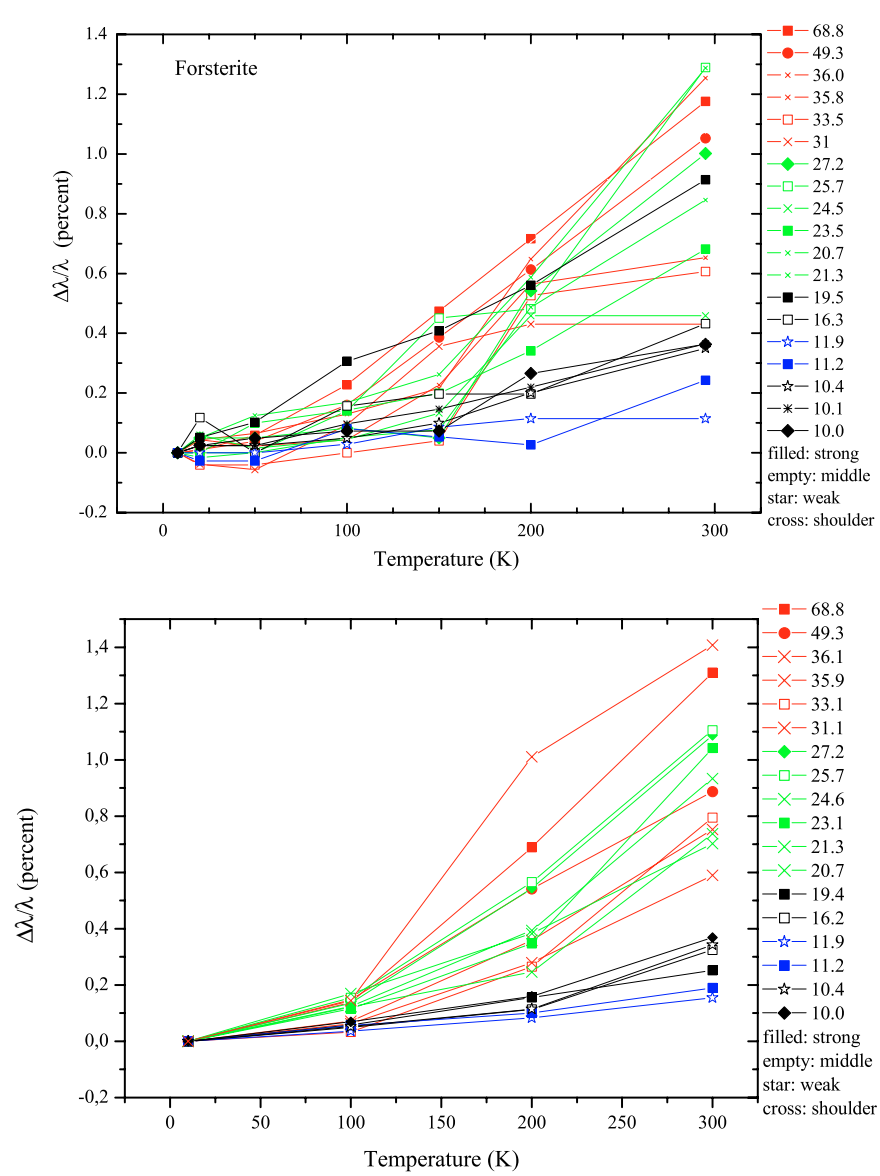

Fig. 4. Temperature-dependence of the relative peak shifts $\Delta \lambda / \lambda\left(T_{0}\right)$ (in \%) for the forsterite bands. Upper and lower panel correspond to Kyoto and Jena measurements, respectively. Here, $\Delta \lambda=\lambda(T)-$ $\lambda\left(T_{0}\right)$, and $T_{0}$ is $8 \mathrm{~K}$ (Kyoto) or $10 \mathrm{~K}$ (Jena). The labels in the legends correspond to $\lambda\left(T_{0}\right)$.

easily ground into very fine particles, which may result in similar grain shapes even with different grinding methods. This hypothesis is supported by new calculations of small-particle Fo spectra based on temperature-dependent optical constants. Suto et al. (in preparation) measured the reflectance spectra of single crystalline forsterite at temperatures down to $50 \mathrm{~K}$ for field directions parallel to each of the crystal axes. They obtained the optical constants by fitting oscillator parameters of a dispersion formula. The mass absorption coefficients calculated from these data for a continuous distribution of ellipsoidal particles (CDE) have been compared for each temperature with the present data of the Kyoto sample, and they generally reproduced the present features well (Naoi et al. 2004). Some discrepancies observed in the comparison could be due to the choice of the CDE shape distribution. As the SEM image of Kyoto forsterite particles reveals a rectangular shape of the particles, the approximation by the elliptical shape could be wrong with shape sensitive features (details in preparation). This indicates that some difference between the Kyoto and Jena data may also be due to shape effects. In fact, the spectra of the Kyoto sample even at room temperature depend a little on individual $\mathrm{KBr}$ and $\mathrm{PE}$ sheets. Forsterite particles are very hard to grind into fine particles, which could lead to slightly different grain shapes in each pellet of $\mathrm{KBr}$ and $\mathrm{PE}$. As shown by Fabian et al. (2001), near-spherically shaped olivine grains (CDE2) show most peak positions at shorter wavelengths than particles of more elongated ellipsoidal shapes (CDE1, corresponds to CDE used by Naoi et al. 2004). Experimentally, Day (1975) measured the infrared spectra of forsterite spheres (diameter around 500-600 А) embedded in $\mathrm{KBr}$, where the positions of strong peaks shifted to even shorter wavelengths compared with the present data. Consequently, the Jena samples may contain particles of a more spherical shape than the Kyoto ones due to grinding with a Sialon ball mill.

\subsection{FWHM and peak positions of 49 and $69 \mu \mathrm{m}$ bands}

As mentioned already, the forsterite 49 and $69 \mu \mathrm{m}$ bands are particularly important for a comparison with observations. Therefore, we took special care to obtain exact band positions and the full width at half-maximum (FWHM). In the farinfrared region, the measured spectra generally show interference fringes due to multiple reflection in the PE sheets and pellets (thickness about $1 \mathrm{~mm}$ ). Since these fringes affect the peak positions, especially for the 49 and $69 \mu \mathrm{m}$ bands, we removed them by subtracting trigonometric functions. The result is shown in Fig. 8 for the Kyoto data. After subtracting a linear baseline, the features have been fitted by Lorentzian profiles in order to obtain the peak positions and FWHM. The corrected peak positions are plotted in Fig. 9. They are a little shifted compared to the original positions that were read by eye. The errors of the peak positions as estimated from the Lorentzian fits, are within $0.010 \mu \mathrm{m}$ for $295 \mathrm{~K}$ and $200 \mathrm{~K}$, and $0.005 \mu \mathrm{m}$ for $150-10 \mathrm{~K}$. The intrinsic FWHM of the bands is calculated from the ones obtained from the Lorentzian fits assuming that the measured feature profiles are convolutions of intrinsic Lorentzian band profiles and Gaussian instrumental response curves. The widths of the latter were adapted from the spectral resolutions used in our measurements. The corrected FWHM for both the Kyoto and Jena samples is plotted in Fig. 10 versus the temperature and the band position. These corrected peak positions and FWHM are also compiled in Table 1.

The FWHM of the 49 and $69 \mu \mathrm{m}$ bands in wavelength $(\mu \mathrm{m})$ are also functions that are sensitive to the temperature and that correlate well with the peak position as shown in Fig. 10. The FWHM actually increases almost linearly with the peak positions, especially for the $69 \mu \mathrm{m}$ band. This is true for both the Kyoto and Jena data. However, Fig. 10 also shows clearly that the FWHM measured for the Jena forsterite sample is significantly larger than the corresponding Kyoto data. This difference is systematic for all temperatures and it has not changed significantly after correcting for the different resolutions in the Kyoto and Jena measurements. Therefore, we assume that this band broadening for the Jena sample is either due to a lower crystal quality of the Jena forsterite or due to particle coagulation. While the Kyoto sample is a single crystal grown by a special growth method, the Jena sample is polycrystalline consisting of microcrystallites in the micron size range (Fabian et al. 2001). The influence of cristallinity on forsterite band profiles has already been discussed by Molster et al. (2002) 


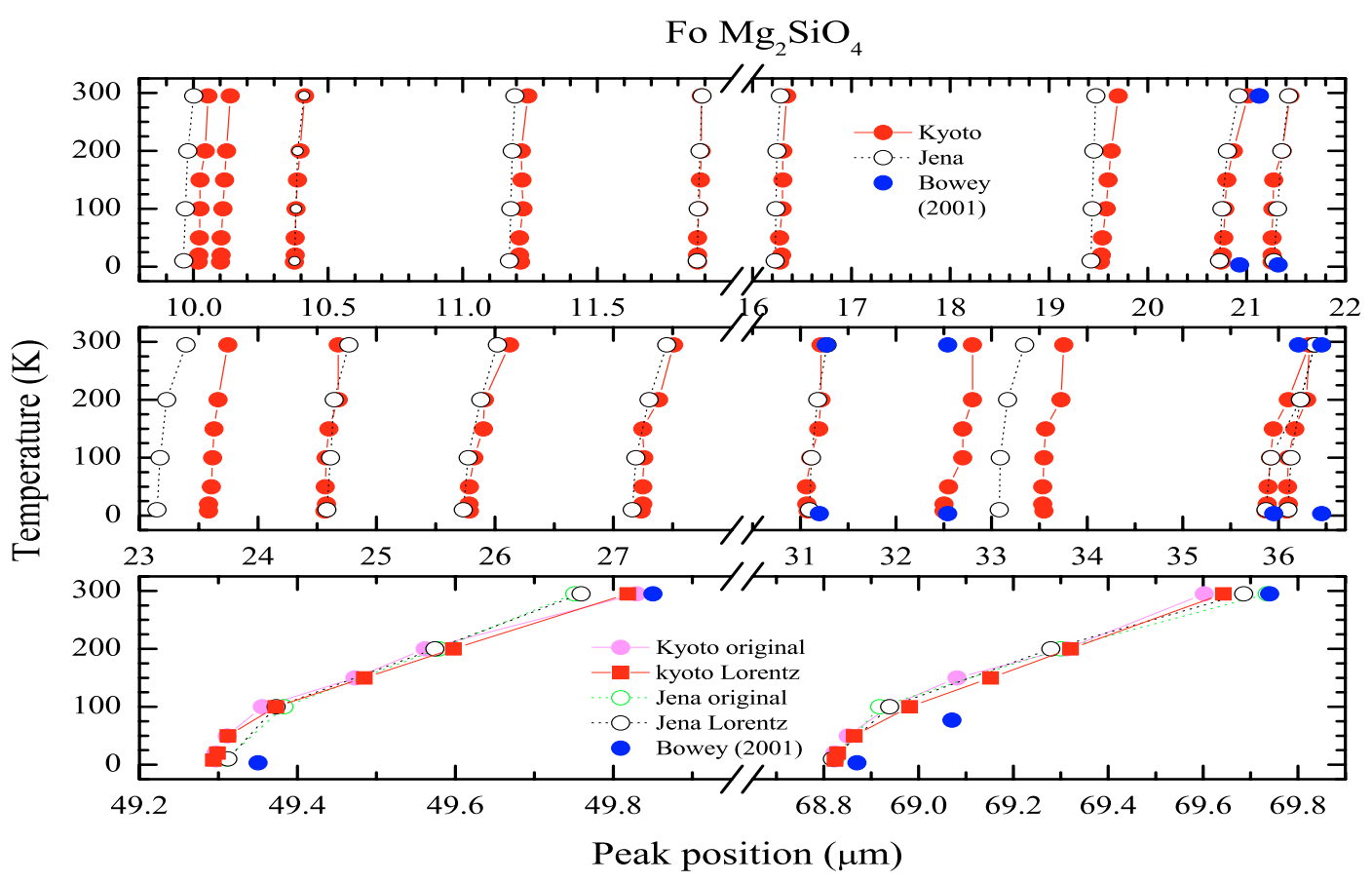

Fig. 5. Temperature-dependence of the peak positions for the forsterite bands (read from the spectra by eye). Full and open symbols correspond to the Kyoto and Jena samples, respectively. For the 49 and $69 \mu \mathrm{m}$ bands, data obtained by Lorentzian fits are also plotted. Data by Bowey et al. (2001) are plotted for comparison.

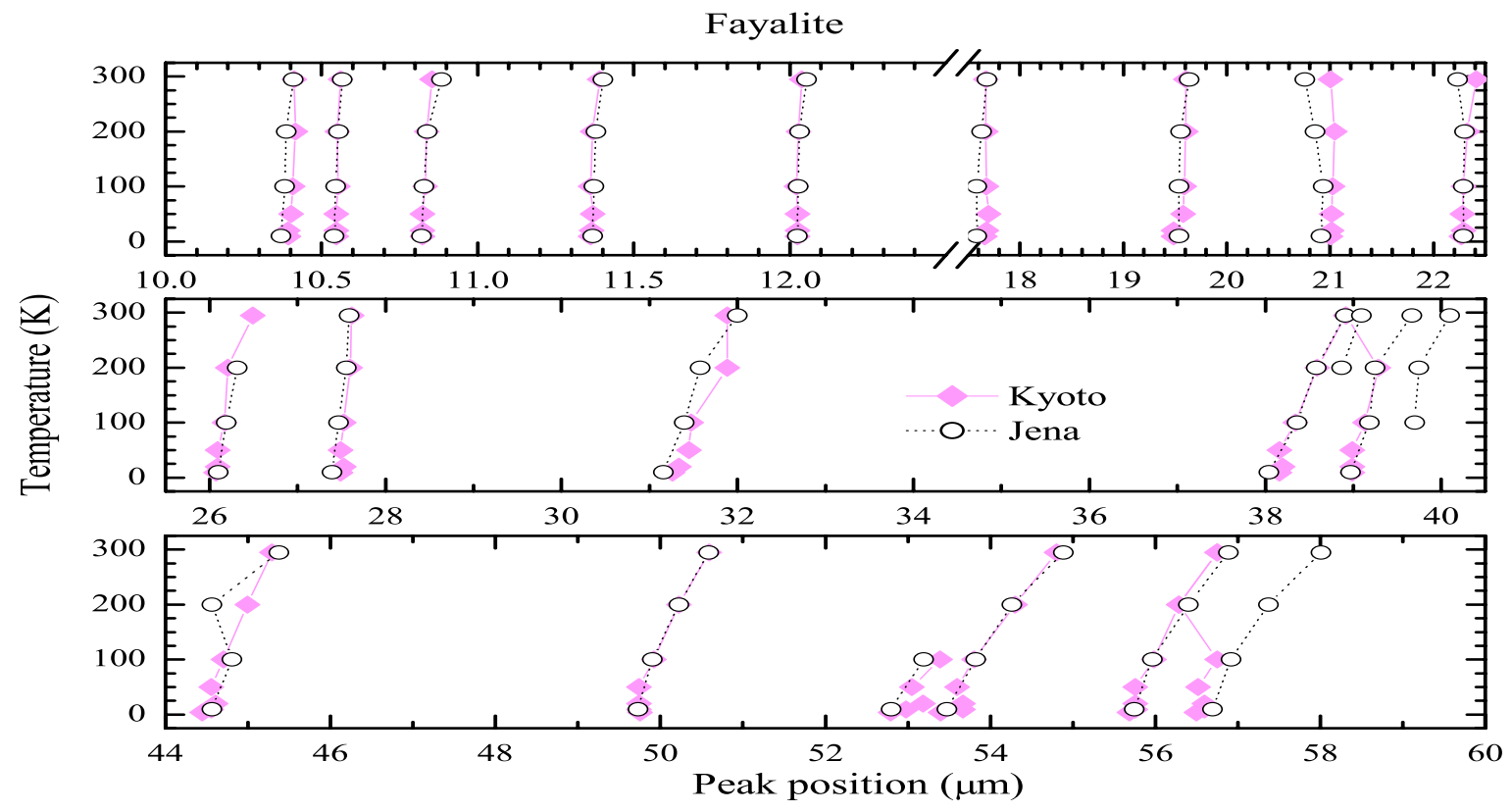

Fig. 6. Temperature-dependence of the peak positions for the fayalite bands (read from the spectra by eye). Full and open symbols correspond to Kyoto and Jena samples, respectively.

in connection with the profile variation in the $33.5 \mu \mathrm{m}$ band, which we now believe is mainly due to shape effects. Recently, the Kyoto group measured synthetic polycrystalline Fo, and these spectra show sharp peaks that are similar to those of single crystal Fo (Koike et al. in preparation). Although this still does not exclude any influence on the FWHM of the crystal quality, because the quality of polycrystalline lattices may of course differ, another possibility for causing differences in
FWHM is particle coagulation, which may strongly affect the sharpness of bands.

In comparing our new data with the Bowey et al. (2001, 2002) data and with Chihara (2001) data shown in Figs. 9 and 10 , the peak positions coincide at room temperature and $3.5 \mathrm{~K}$ or $4.2 \mathrm{~K}$. Small deviations may be due to shape effects or particle aggregation. However, at $77 \mathrm{~K}$ there is a significant difference in the position of the $69 \mu \mathrm{m}$ band for 


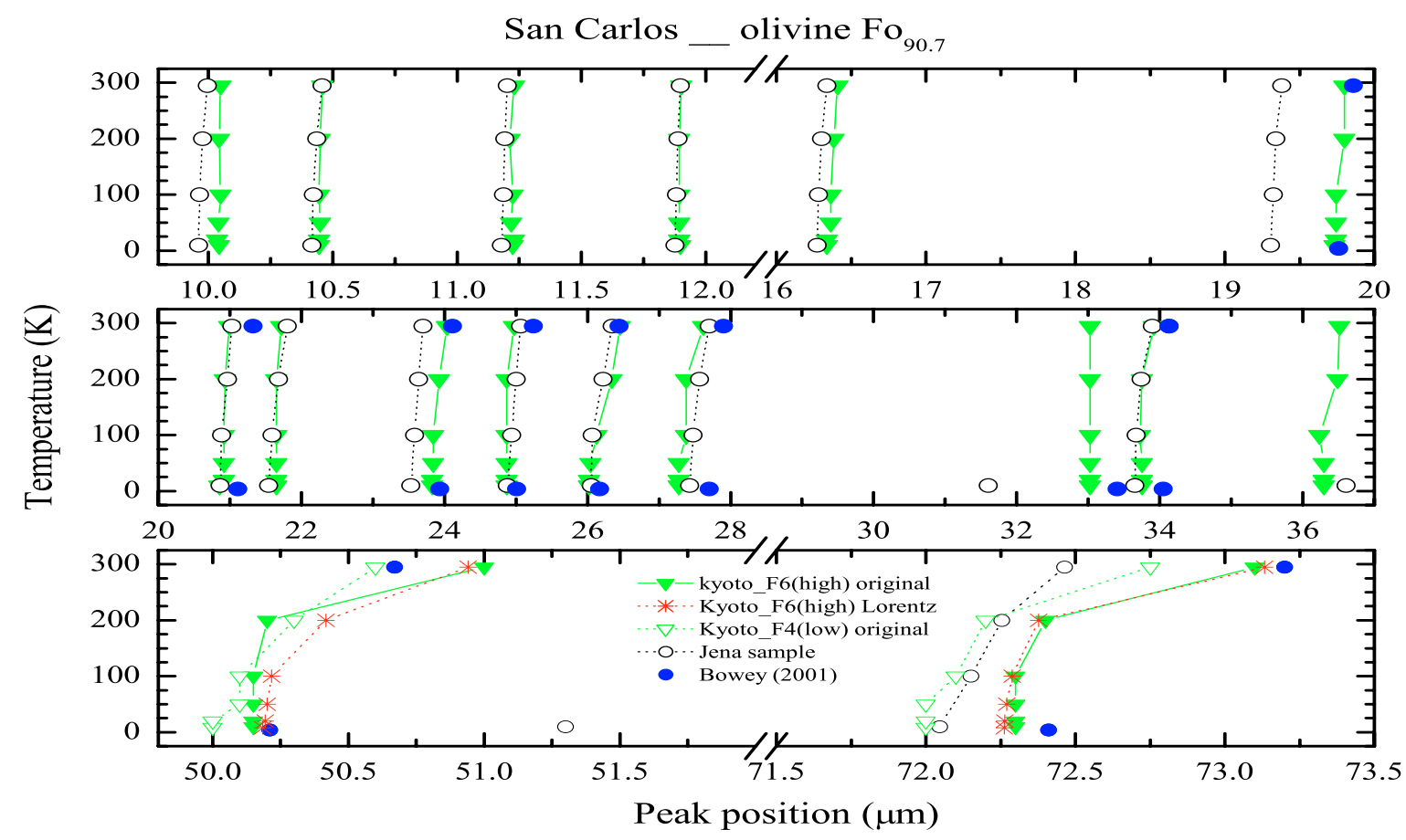

Fig. 7. Temperature-dependence of the peak positions for the San Carlos olivine bands (read from the spectra by eye). Full and open symbols correspond to Kyoto and Jena samples, respectively. Data by Bowey et al. (2001) are plotted for comparison.

both the Kyoto and Jena data compared to that of Bowey et al. (2001, 2002). From our new results (see Fig. 9), it has to be concluded that at $77 \mathrm{~K}$ the peak position of this band is at a wavelength positions that is shorter by about $0.2 \mu \mathrm{m}$. This is an important point that will actually change the dust temperatures derived from observed spectra dramatically, as we show. Also, the FWHM measured by Bowey et al. for the $69 \mu \mathrm{m}$ peak at $77 \mathrm{~K}$ is somewhat larger than would have been expected from the FWHMs at room temperature and $3.5 \mathrm{~K}$. These two are well between the Kyoto and Jena data and nearly coincide with Chihara's (2001) data (see lower right panel of Fig. 10). The deviation in both position and FWHM, which however maintains the linear correlation between both parameters (see upper right panel of Fig. 10), may simply point to an actually higher sample temperature in the $77 \mathrm{~K}$ measurement by Bowey et al. $(2001)^{2}$.

\subsection{Comparison with observations}

As for the $69 \mu \mathrm{m}$ band, an emission feature in circumstellar spectra at $69 \mu \mathrm{m}$ observed with ISO has been identified with nearly pure Mg-olivine particles (Malfait et al. 1998). The peak positions and widths of this $69 \mu \mathrm{m}$ band have been analysed for

\footnotetext{
2 A simple estimate from the band position (Fig. 9) would result in a sample temperature of about $120 \mathrm{~K}$ instead of $77 \mathrm{~K}$ for the Bowey et al. measurement. This temperature modification would also shift their FWHM between the Jena and Kyoto data (Fig. 10). According to a private communiation by $\mathrm{J}$. Bowey, the temperature conditions in their experiment were quite unstable when using the cold finger at liquid-nitrogen temperature. This may explain the discrepancy between theirs and our data.
}

several objects by Bowey et al. (2002) and Molster et al. (2002). As an example, we plot their data (FWHM vs. peak position) for four objects in Fig. 11 (lower panel), together with the available temperature-dependent laboratory data. The observational data cover a range of $68.8 \mu \mathrm{m}$ and $69.3 \mu \mathrm{m}$ in peak position and between $0.3 \mu \mathrm{m}$ and $0.8 \mu \mathrm{m}$ in FWHM. The same range is covered by the laboratory data at grain temperatures below $200 \mathrm{~K}$, with the Jena data being at higher FWHM but identical peak positions, as discussed in Sect. 4.2. It is noteworthy that there is also a systematic difference between the observational data obtained by Molster et al. (2002) and by Bowey et al. (2002), where the former in general have somewhat larger FWHM and lower peak position. This may point to a difference in the data evaluation procedure (see the original papers).

Bowey et al. (2002) have determined forsterite grain temperatures for the observed objects from the peak positions of the $69 \mu \mathrm{m}$ band using their laboratory data. Molster et al. (2002) also derive forsterite grain temperatures, but by fitting the emission spectra in the wavelength interval between 5 and $50 \mu \mathrm{m}$, especially the 23 and $33 \mu \mathrm{m}$ complexes, without using temperature-dependent material data. We plot these temperatures derived by both authors in Fig. 11, upper panel, versus the $69 \mu \mathrm{m}$ band position, together with the laboratory-obtained peak positions. The graph shows clearly that, when using our new data instead of the interpolation between the previous data, the forsterite temperatures resulting from the Bowey et al. (2002) observations would have to be increased substantially. For two of the objects (NGC 6537 and HD 44179), the forsterite temperature would then correspond quite well to the temperatures derived by Molster et al. (2002). For the two other objects it would be higher than these, especially for MWC 922, 
Table 1. Peak positions of each band of Fo for the Kyoto and the Jena sample (in $\mu \mathrm{m}$ ).

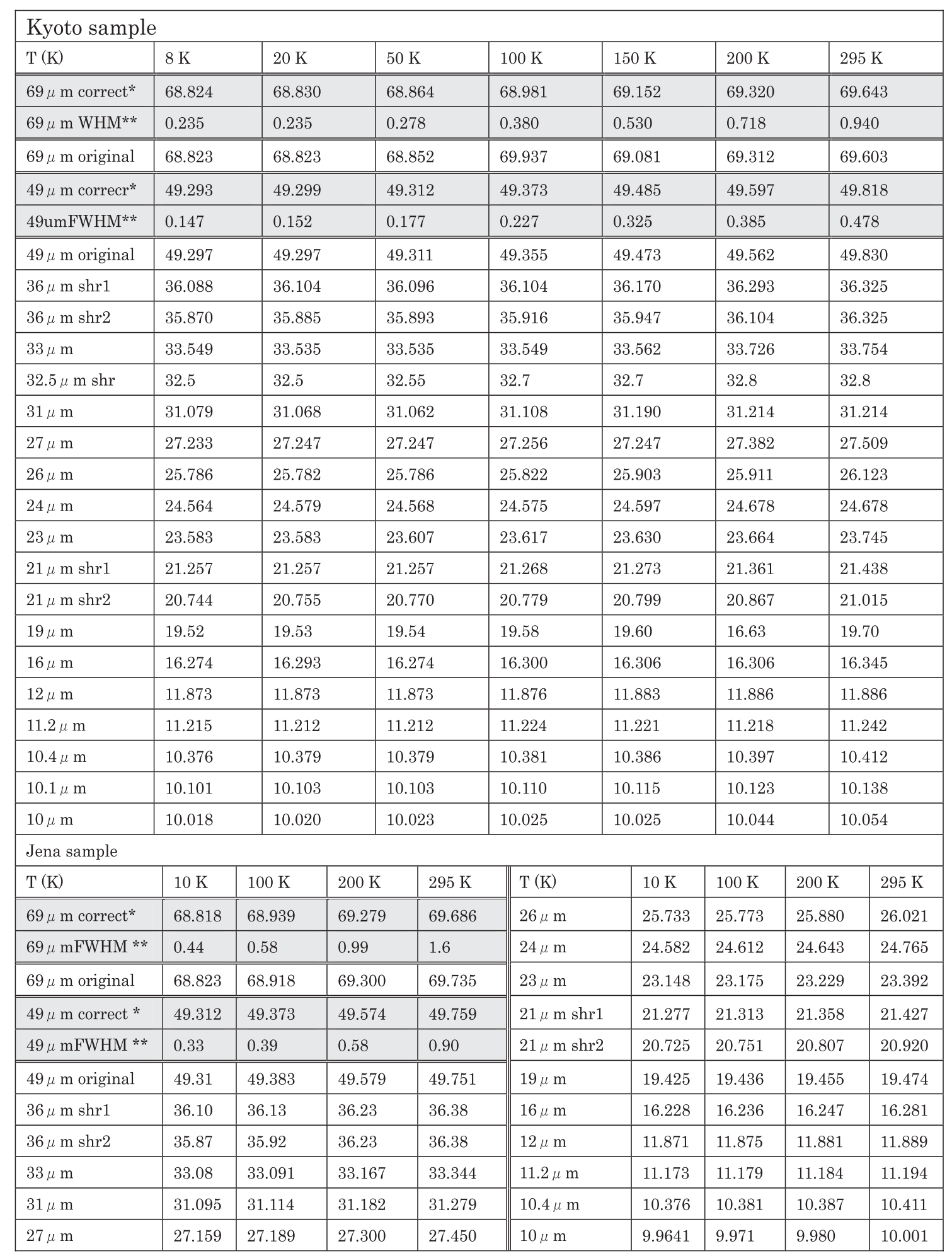

original; read with eye shr; shoulder

* ; fringe correction and Lorentzian $\quad * *$; fringe correction, Lorentzian and instrumental correction

where the fit of the 5 to $50 \mu \mathrm{m}$ spectrum by Molster et al. results in a forsterite temperature of $90 \mathrm{~K}$ (see Fig. 11, upper panel) compared to $170 \mathrm{~K}$ following from the $69 \mu \mathrm{m}$ band position and our data. (Bowey et al. 2002, found $139 \mathrm{~K}$, and interpreted the relatively high grain temperature in this premain sequence object as the possible consequence of the existence of a hotter, optically thin layer above a disk or torus.) This is difficult to explain because it is not expected that 


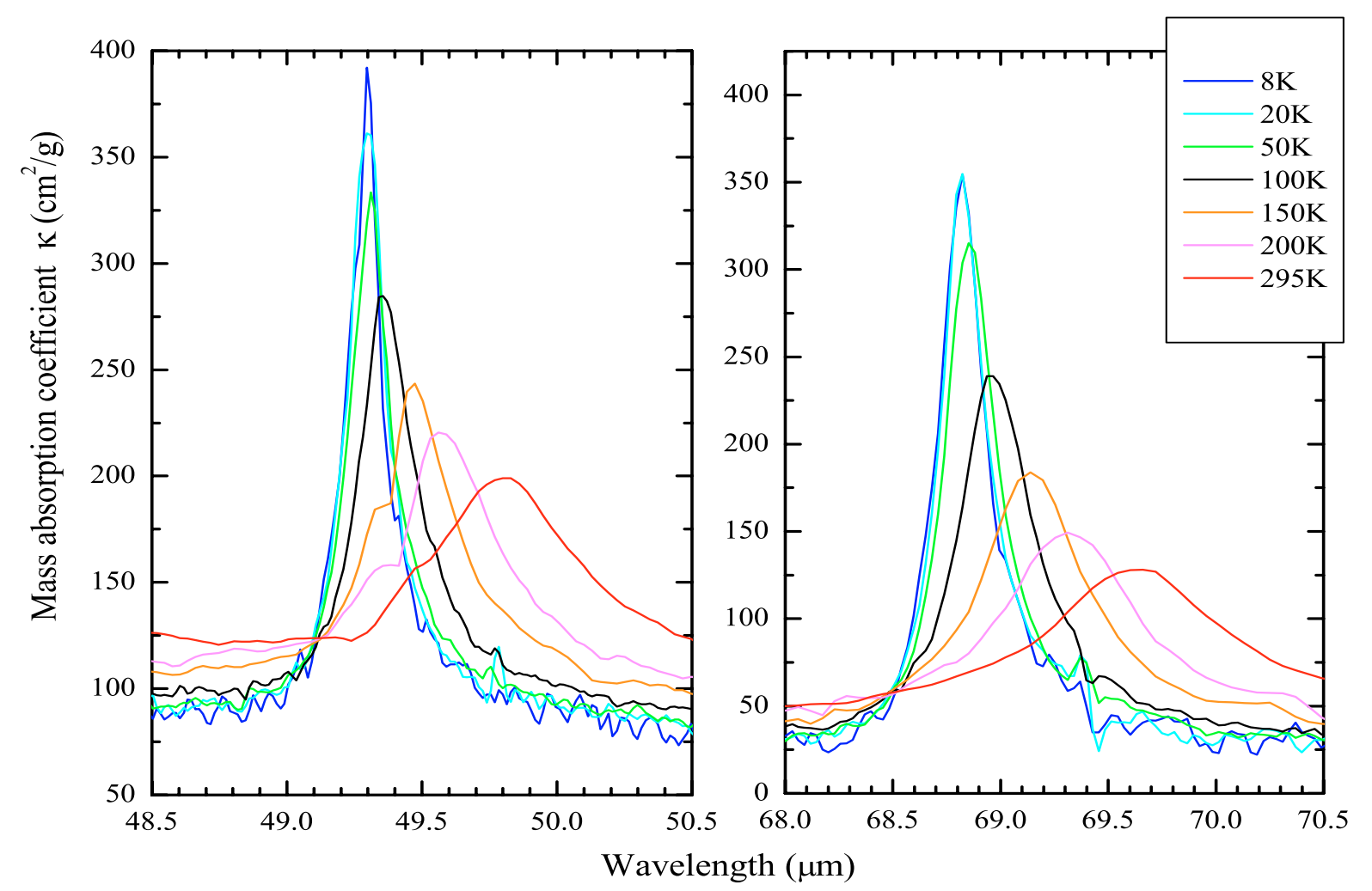

Fig. 8. The band profiles of the 49 and $69 \mu \mathrm{m}$ forsterite bands after fringe correction for the Kyoto sample.

the forsterite grains emitting at $69 \mu \mathrm{m}$ should be warmer than those emitting at shorter wavelengths. Although we note that the amorphous silicate dust component in this object, according to Molster et al., also has a relatively high temperature of $140 \mathrm{~K}$, they state that the fit for MWC 922 is one of the best, so that there is no obvious reason not to trust the forsterite temperature of $90 \mathrm{~K}$. Consequently, we have to consider the possible influence of non-thermal factors on the band position as possible reasons for the higher temperature obtained from the $69 \mu \mathrm{m}$ band position.

First of all, there is the iron content of olivine particles, which heavily effects the peak position (see also Molster et al. 2002). A comprehensive study of the effect of the iron content on peak positions of olivine bands has been published by Koike et al. (2003). They find a linear dependence of the peak position (in wavenumbers) on the forsterite content. According to this rule, a band shift by $0.1 \mu \mathrm{m}$ would correspond to a change in iron content of approximately $0.3-0.5 \%$, although in the range of iron content smaller than $5 \%$ there are no experimental data available yet. Crystal defects should preferentially affect the band width as discussed above and not so much the peak position. Shape and matrix effects are assumed to be not important for the $69 \mu \mathrm{m}$ band compared to the very strong 23.5 and $33.5 \mu \mathrm{m}$ bands. However, this will only be clarified with temperature-dependent optical constants (Suto et al., in preparation).

Future studies of grain temperatures will certainly also benefit from new observations at higher $\mathrm{S} / \mathrm{N}$ ratios. Then it should be possible to use also other bands as temperature indicators such as the forsterite $49 \mu \mathrm{m}$ band, which was hardly detected previously due to the strong continuum (Bowey et al. 2002). Our new laboratory data are a step in this direction. From both better observations and laboratory data, it may be possible to determine the dust temperature more accurately by comparing the observed peak position and FWHM with the laboratory spectra.

\section{Conclusions}

We have quantified the development of the peak positions and, also for some bands of the FWHM in the absorption spectra of olivine particles (forsterite, fayalite, and natural olivine) when cooling from room temperature to $10 \mathrm{~K}$. All spectra depend on temperature. When the temperature decreases, the main effects on the peaks can be summarized as follows:

1. The peak positions shift to shorter wavelength.

2. The shift per temperature interval is often smaller at temperatures below $100 \mathrm{~K}$ than at higher temperatures.

3. The intensities of the peaks increase.

4. The peaks become sharper. For the forsterite $69 \mu \mathrm{m}$ band, the change in width is approximately proportional to the change in peak position.

These tendencies can be seen particularly well for the bands in the far-infrared region. The tendencies are almost identical in those spectra measured on different samples in Kyoto and Jena. For the peak positions, some differences between the Kyoto and Jena samples may be mainly due to shape effects. 


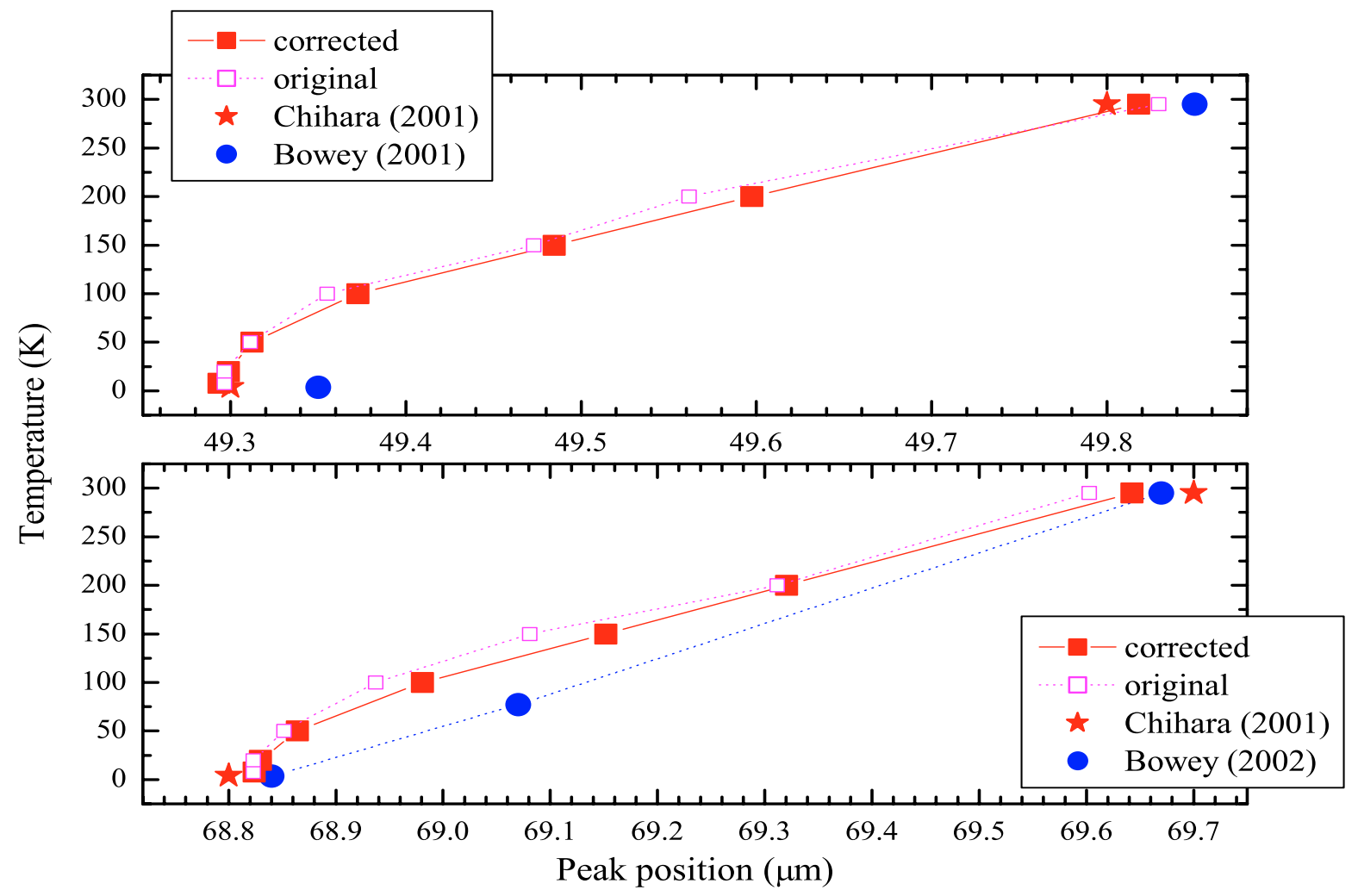

Fig. 9. Temperature dependence of the peak positions for the 49 and $69 \mu \mathrm{m}$ forsterite bands (in $\mu \mathrm{m}$ ) after fringe correction.
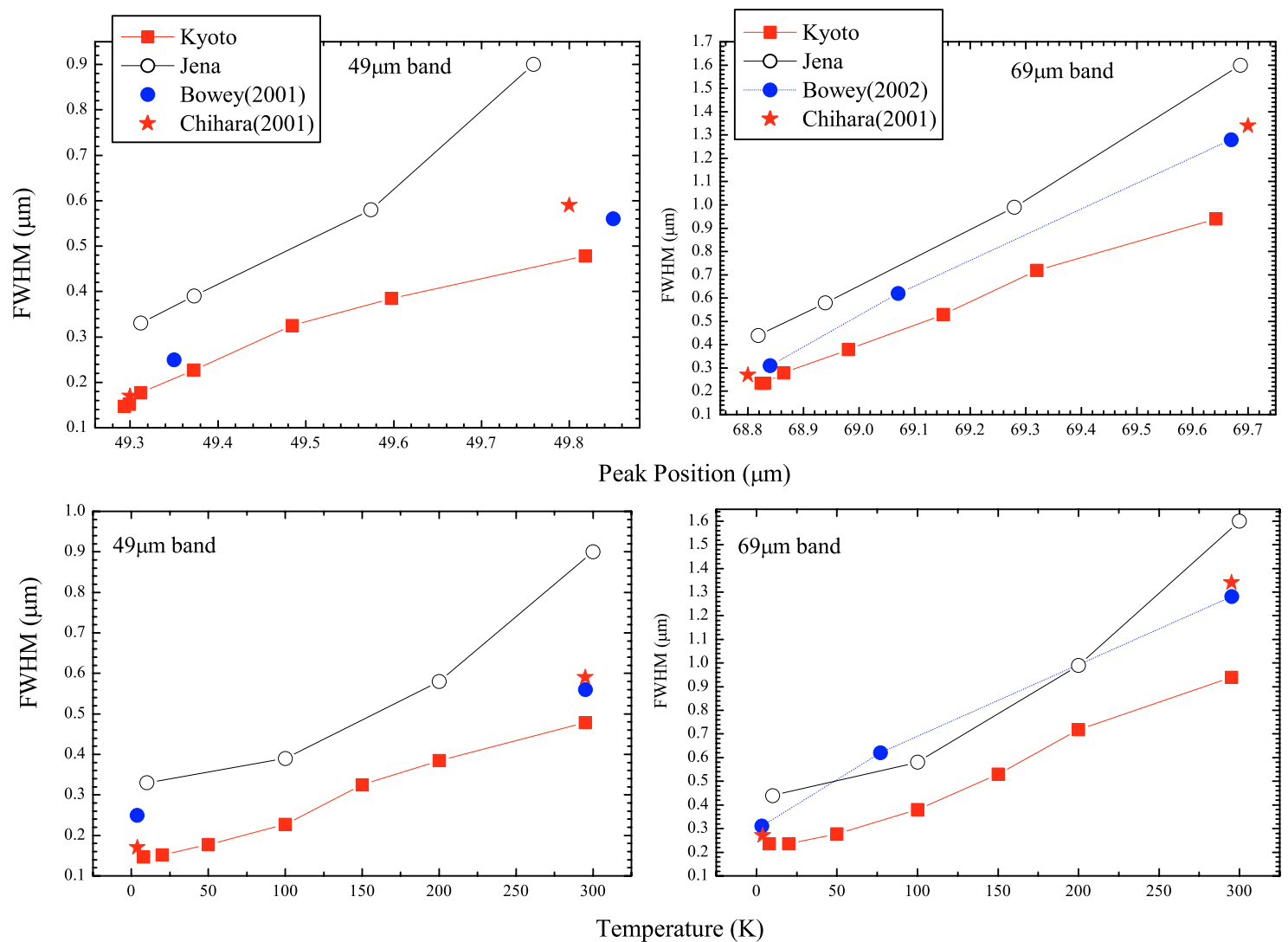

Fig. 10. Temperature and peak-position dependence of the FWHM for the 49 and $69 \mu \mathrm{m}$ forsterite bands after fringe correction and instrument correction. Open circles are for the Jena sample. Data by Chihara $(2001)$ and Bowey $(2001,2002)$ are plotted for comparison. 

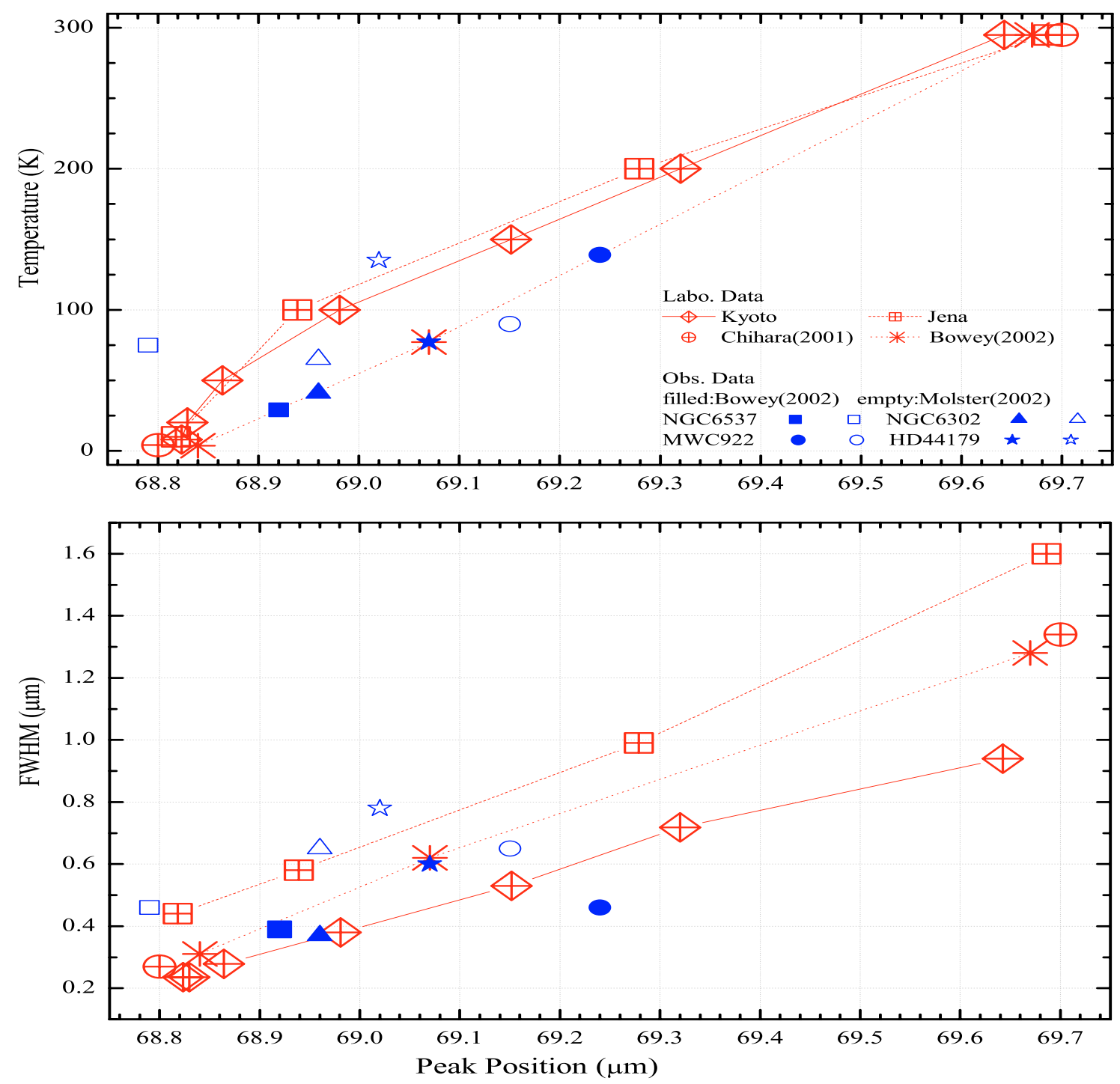

Fig. 11. Comparison of the temperature-dependent peak positions and FWHM (Kyoto and Jena samples after instrumental correction) of the forsterite $69 \mu \mathrm{m}$ band with observations. Data by Chihara et al. (2001) and Bowey et al. (2002) are also plotted. In both panels, FWHM ( $\mu \mathrm{m}$ ) and temperatures derived from observations with model fitting by Bowey et al. (2002) and Molster et al. (2002) are plotted for comparison.

Further differences in the band widths may have been caused by the crystal quality and particle coagulation of the samples. The new data allow a significantly more accurate determination of olivine dust temperatures from observational spectra.

Acknowledgements. The Kyoto group was supported by Grantsin-Aid from the Japanese Ministry of Education, Culture, Sports, Science, and Technology (12440054 and 15340064), and by MEXT Japan, Grant-in-Aid for Scientific Research on Priority Areas, "Development of Extra-Solar Planetary Science" (17039006). They were also supported by the National Astronomical Observatory of Japan. The Jena group was supported by DFG grants Do 575/4 and Mu 1164/5. They wish to thank W. Teuschel for major help with the measurements, G. Born for sample preparation, and W. Assmus, Univ. Frankfurt/M. for supplying the fayalite crystals. The collaboration between Jena and Heidelberg is supported by a joint initiative in laboratory astrophysics. We would like to thank the referee, Dr. J. E. Bowey, for her constructive and helpful reports, which have improved this paper.

\section{References}

Agladze, N. I., Sievers, A. J., Jones, S. A., Burlitch, J. M., \& Beckwith, S. V. W. 1996, ApJ, 462, 1026

Boudet, N., Mutschke, H., Nayral, C., et al. 2005, ApJ, in press

Bowey, J. E., Barlow, M. J., Molster, F. J., et al. 2002, MNRAS, 331, L1

Bowey, J. E., Lee, C., Tucker, C., et al. 2001, MNRAS, 325, 886

Chihara, H., Koike, C., \& Tsuchiyama, A. 2001, Publ. Astron. Soc. Jpn., 53, 243

Colangeli, L., Henning, Th., Brucato, J. R., et al. 2003, A\&ARv, 11, 97

Crovisier, J., Leech, K., Bochelee-Morvan, D., et al. 1997, Science, 275,1904

Day, K. L. 1975, ApJ, 199, 660

Day, K. L. 1976, ApJ, 203, L99

Fabian, D., Henning, Th., Jäger, C., et al. 2001, A\&A, 378, 228

Henning, Th. 2003, in Solid-State Astrochemistry, ed. V. Pirronello, J. Krelowski, \& L. Manico (Dordrecht: Kluwer), 85 
Henning, Th., \& Mutschke, H. 1997, A\&A, 327, 743

Hofmeister, A. 1997, Phys. Chem. Min., 24, 535

Jäger, C., Molster, F. J., Dorschner, J., et al. 1998, A\&A, 339, 904

Koike, C., Chihara, H., Tsuchiyama, A., Sogawa, H., \& Suto, H. 2000, Proc. of the 33rd ISAS Lunar and Planetary Symp., 33, 95

Koike, C., Chihara, H., Tsuchiyama, A., et al. 2003, A\&A, 399, 1101

Malfait, K., Waelkens, C., Waters, L. B. F. M., et al. 1998, A\&A, 332, L25

Malfait, K., Waelkens, C., Bouwman, J., de Koter, A., \& Waters, L. B. F. M. 1999, A\&A, 345, 181

Mennella, V., Brucato, J. R., Colangeli, L., et al. 1998, ApJ, 496, 1058

Mitra, S. S. 1985, in Handbook of Optical Constants of Solids, ed. E. D. Palik (Orlando: Academic Press), 213
Molster, F. J., Waters, L. B. F. M., \& Tielens, A. G. G. M. 2002, A\&A, 382,241

Naoi, T., Koike, C., Suto, H., et al. 2004, Proc. 37th ISAS Lunar and Planetary Symp., ed. H. Mizutani, \& M. Kato, 99

Posch, Th., Kerschbaum, F., Richter, H., \& Mutschke, H. 2005, Proc. The Dusty and Molecular Universe, ed. A. Wilson, ESA SP-577, 257

Waters, L. B. F. M., Beintem, D. A., Zijlstra, A. A., et al. 1998, A\&A, 331, L61

Waelkens, C., Waters, L. B. F. M., de Grauw, M. S., et al. 1996, A\&A, 315, L245

Zaikowski, A., Knacke, R. F., \& Porco, C. C. 1975, Ap\&SS, 35, 97 